\title{
Characterization of universal two-qubit Hamiltonians
}

\author{
Andrew M. Childs, Debbie Leung, Laura Mancinska, and Maris Ozols \\ Department of Combinatorics \& Optimization \\ and Institute for Quantum Computing \\ University of Waterloo
}

\begin{abstract}
Suppose we can apply a given 2-qubit Hamiltonian $H$ to any (ordered) pair of qubits. We say $H$ is $n$-universal if it can be used to approximate any unitary operation on $n$ qubits. While it is well known that almost any 2-qubit Hamiltonian is 2-universal (Deutsch, Barenco, Ekert 1995; Lloyd 1995), an explicit characterization of the set of non-universal 2-qubit Hamiltonians has been elusive. Our main result is a complete characterization of 2-non-universal 2-qubit Hamiltonians. In particular, there are three ways that a 2-qubit Hamiltonian $H$ can fail to be universal: (1) $H$ shares an eigenvector with the gate that swaps two qubits, (2) $H$ acts on the two qubits independently (in any of a certain family of bases), or (3) $H$ has zero trace. A 2-non-universal 2-qubit Hamiltonian can still be $n$-universal for some $n \geq 3$. We give some partial results on 3-universality. Finally, we also show how our characterization of 2-universal Hamiltonians implies the well-known result that almost any 2-qubit unitary is universal.
\end{abstract}

\section{Introduction}

It is often useful to understand when a given set of resources is sufficient to perform universal computation. In particular, universal Hamiltonians have many applications in quantum computation.

Suppose we can implement one specific 2-qubit Hamiltonian $H \in \mathfrak{u}(4)$, where $\mathfrak{u}(4)$ denotes the set of all $4 \times 4$ Hermitian matrices. Assume we have $n$ qubits and we can apply $H$ to any ordered pair of them for any amount of time. We say that $H$ is $n$-universal if it is possible to approximate any unitary evolution $U \in \mathrm{U}\left(2^{n}\right)$ to any desired accuracy by repeatedly applying $H$ to different pairs of qubits.

It is known that almost any 2-qubit Hamiltonian is universal [8, 14, i.e., non-universal 2-qubit Hamiltonians form a measure-zero subset of $\mathfrak{u}(4)$. Thus generic interactions are suitable for universal computation. But this does not address the issue of deciding whether a particular Hamiltonian is universal.

Given a specific $H \in \mathfrak{u}(4)$, one can check numerically if $H$ is $n$-universal by determining whether $H$, when applied on different pairs of qubits, generates the Lie algebra of $\mathrm{U}\left(2^{n}\right)$ (see Section 2.4. However, this characterization can be inconvenient for answering structural questions about universality. For example, suppose we can experimentally implement Hamiltonians of a certain restricted form, say, $\alpha(X \otimes I)+\beta(Y \otimes Y)$ for some $\alpha, \beta \in \mathbb{R}$. Determining which of these Hamiltonians are universal is not straightforward using the Lie-algebraic characterization. Indeed, until now there has been no simple closed-form characterization of the set of non-universal 2-qubit Hamiltonians.

In this paper we characterize the set of all 2-non-universal 2-qubit Hamiltonians. In particular, our characterization easily answers questions such as those described above. We give a finite list of families of 2-non-universal 2-qubit Hamiltonians such that each family can be easily parametrized and together they cover all 2-non-universal 2-qubit Hamiltonians.

The remainder of the paper is organized as follows. Section 2 introduces the concept of universality. We give our definition of universality, contrast this definition with some alternatives, review previous related work, and present a Lie-algebraic formulation. Section 3 then establishes our main result. We start from some simple families of Hamiltonians that are obviously 2-non-universal, extend them with a class of operations that preserve this property, and then show that the extended families exactly characterize 2-universality. Section 4 briefly summarizes what we know about 3-universality. Finally, we conclude in Section 5 with a discussion of some open problems. 
In Appendix D (added after this paper was published), we show how our results easily imply the wellknown result that almost any 2-qubit unitary is universal.

\section{Universality in quantum computing}

\subsection{Definition of universality}

We begin with some basic definitions needed to precisely specify the problem addressed by this paper.

Definition 1. We say that $H$ is an n-qubit Hamiltonian if $H \in \mathfrak{u}\left(2^{n}\right)$, i.e., $H \in \mathrm{M}_{2^{n}}(\mathbb{C})\left(\mathrm{M}_{N}(\mathbb{C})\right.$ denotes the set of $N \times N$ complex matrices) and $H$ is Hermitian $\left(H^{\dagger}=H\right)$.

In this paper we mainly deal with 2 -qubit Hamiltonians, i.e., $4 \times 4$ Hermitian matrices. We often say "a Hamiltonian $H$ " without explicitly mentioning that it is a 2-qubit Hamiltonian.

Definition 2. We say that we can simulate a unitary transformation $U \in \mathrm{U}(N)$ using Hamiltonians $H_{1}, \ldots, H_{k} \in \mathfrak{u}(N)$ if for all $\varepsilon>0$ there exist $l \in \mathbb{N}, j_{1}, \ldots, j_{l} \in\{1, \ldots, k\}$, and $t_{1}, \ldots, t_{l}>0$ such that

$$
\left\|U-e^{-i H_{j_{1}} t_{1}} e^{-i H_{j_{2}} t_{2}} \ldots e^{-i H_{j_{l}} t_{l}}\right\|_{\infty}<\varepsilon
$$

Definition 2 only allows the use of positive $t_{i}$ for simulating a unitary $U$ by Hamiltonians $H_{1}, \ldots, H_{k}$, since $t_{i}$ corresponds to the length of time the system evolves according to $H_{j_{i}}$. However, this restriction can be relaxed to $t_{i} \in \mathbb{R}$. This is because an evolution by negative time can be approximated by evolving our system according to $H$ for some positive time instead (see Claim 1 in Appendix A for a proof).

We only require the ability to approximate any unitary to arbitrary precision. Such a definition is motivated by related universality problems based on discrete universal gate sets to be discussed below. We are not concerned about the time it takes to complete the simulation as long as we can simulate any unitary. Also, we do not assume the availability of ancillary systems.

Definition 3. Let $2 \leq m \leq n$. We say that an $m$-qubit Hamiltonian $H$ is $n$-universal if we can simulate all unitary transformations in $\mathrm{U}\left(2^{n}\right)$ using Hamiltonians from the set

$$
\left\{P\left(H \otimes I^{\otimes n-m}\right) P^{\dagger} \mid P \in \mathcal{S}_{n}\right\},
$$

where $\mathcal{S}_{n}$ is the group of matrices that permute $n$ qubits. That is, we can apply $H$ to any ordered subset of $m$ qubits (out of $n$ qubits in total).

The main goal of this paper is to characterize the set of 2-universal 2-qubit Hamiltonians. One motivation for this is that any 2-universal 2-qubit Hamiltonian is also $n$-universal for all integers $n \geq 2$ (see Lemma 2 in Section 2.4). Note that a 2-qubit Hamiltonian $H$ is 2-universal if we can simulate all unitary transformations in $\mathrm{U}(4)$ using $H$ and $T H T$, where $T$ is the gate that swaps the two qubits, with the following representation in the computational (i.e., standard) basis:

$$
T:=\left(\begin{array}{cccc}
1 & 0 & 0 & 0 \\
0 & 0 & 1 & 0 \\
0 & 1 & 0 & 0 \\
0 & 0 & 0 & 1
\end{array}\right) .
$$

To achieve our goal, we classify those 2-qubit Hamiltonians that are not 2-universal.

\subsection{Other notions of universality}

Universal primitives for quantum computation, such as Hamiltonians and unitary gates, have been extensively studied previously; see for example Refs. 1, 2, 7, 8, 9, 13, 14, 18. Since the primitives are often physically motivated, there are different definitions of universality appropriate for different circumstances. First, one can study the universality of a set of quantum gates (instead of Hamiltonians). Second, one can study universality assuming ancillary qubits can be prepared and used to facilitate the computation. In particular, one might consider the following definitions of universality with ancillae: 
Definition 4. For all $n, k \in \mathbb{N}$ let $\mathcal{C}(n, k)$ be the set of all functions from $n$-bit strings to $k$-bit strings. We say that a set of logical gates $\mathcal{S}$ is classically universal with ancillae if for all $n, k \in \mathbb{N}$ and all $C \in \mathcal{C}(n, k)$ there exist $n_{a} \in \mathbb{N}$ and a logical circuit $G \in \mathcal{C}\left(n+n_{a}, k+n_{a}\right)$ containing gates exclusively from $\mathcal{S}$ that simulates $C$ using ancillae, i.e., there exists $a \in\{0,1\}^{n_{a}}$ such that for all $\psi \in\{0,1\}^{n}$ we have $(C(\psi), a)=G(\psi, a)$.

Definition 5. We say that a set of unitary gates $S$ is (quantumly) universal with ancillae if for all $n \in \mathbb{N}$, all $\varepsilon>0$, and all $U \in \mathrm{U}\left(2^{n}\right)$, there exist $n_{a} \in \mathbb{N}$ and a quantum circuit $G \in \mathrm{U}\left(2^{n+n_{a}}\right)$ containing gates exclusively from $S$ that approximates $U$ with precision $\varepsilon$ using ancillae, i.e., there exists $a \in\{0,1\}^{n_{a}}$ such that for all $|\psi\rangle \in \mathbb{C}^{2^{n}}$ we have $\|(U|\psi\rangle) \otimes|a\rangle-G(|\psi\rangle \otimes|a\rangle) \|<\varepsilon$.

Note that in the above definitions we assume the ability to prepare standard basis states. We allow initializing the ancillary bits to arbitrary standard basis states (as opposed to only $|0\rangle$ ) since some of the gates considered below (e.g., the Toffoli gate and Deutsch's gate) need ancillary bits prepared in basis states other than $|0\rangle$ to achieve universality. However, other reasonable definitions of universality with ancillae are possible. (For example, the ancillary state need not be preserved in Definitions 4 and 5 )

In the classical case we can implement any logical gate exactly using elements of a universal gate set. In contrast, in the quantum case we only require the ability to approximate any unitary to arbitrary precision. This definition is motivated by the need to use discrete universal gate sets to perform fault-tolerant quantum computing [4, 17]; such sets cannot implement a continuum of operations exactly.

\subsection{Previous results}

\subsubsection{Universal gate sets with ancillae}

It is well known that the gate set $\{$ NAND, FANOUT $\}$ is classically universal with ancillae. Deutsch [7] showed that any gate from a certain family of 3-qubit unitary gates is quantumly universal with ancillae. DiVincenzo [9] suggested that it might be difficult to implement Deutsch's unitary gates as it is hard to build a mechanical device that brings three spins together. To obviate this, he devised a set of four 2-qubit unitary gates that is quantumly universal with ancillae. Barenco [1] improved DiVincenzo's result by showing that a single 2-qubit unitary gate $A(\phi, \beta, \theta)$ is universal with ancillae, where

$$
A(\phi, \beta, \theta):=\left(\begin{array}{cccc}
1 & 0 & 0 & 0 \\
0 & 1 & 0 & 0 \\
0 & 0 & e^{i \beta} \cos \theta & -i e^{i(\beta-\phi)} \sin \theta \\
0 & 0 & -i e^{i(\beta+\phi)} \sin \theta & e^{i \beta} \cos \theta
\end{array}\right)
$$

and $\phi, \beta$, and $\theta$ are irrational multiples of $\pi$ and of each other.

\subsubsection{Universal gate sets without ancillae}

Sets of unitary gates have also been found that can approximate any unitary transformation without the use of ancillary qubits. It is well known [6, 16] that the Controlled-NOT gate together with all 1-qubit gates form a universal gate set. Furthermore, several different finite sets of universal 2-qubit quantum gates are known [4, 13, 20.

\subsubsection{Universality of a single 2-qubit gate without ancillae}

In 1995, Deutsch, Barenco, and Ekert [8] and Lloyd [14 independently showed that almost any 2-qubit gate can be used to approximate all 2-qubit unitary evolutions. In other words, the set of non-universal unitary gates forms a measure-zero subset of the group U(4). Notably, in order to achieve universality, ancillary qubits are not required. The approaches used in [8] and [14] are similar in many respects and build upon the Lie-algebraic approach of DiVincenzo [9. Neither approach is constructive and both analyses revolve around the Lie algebra generated by $H$ and $T H T$, where $H$ is a Hamiltonian corresponding to a generic unitary and $T$ is the gate exchanging the two qubits (recall equation (33). The proof in Ref. [14] omits some details (some of which were later filled in by Weaver [21]), whereas Ref. [8] provides a more complete proof.

Our work builds upon some of the techniques described in Ref. [8]. Unfortunately, the arguments of that paper have some shortcomings: 
1. The goal of $[8$ is to establish the universality of a generic unitary $U \in \mathrm{U}(4)$. The argument begins by replacing $U$ with a "Hamiltonian $H$ generating $U$," defined as a solution to $U=e^{i H}$. However, there can be different solutions generating different Lie algebras. As a simple example, both

$$
H:=\left(\begin{array}{llll}
0 & 0 & 0 & 0 \\
0 & 0 & 0 & 0 \\
0 & 0 & 0 & 0 \\
0 & 0 & 0 & 0
\end{array}\right) \quad \text { and } \quad H^{\prime}:=\left(\begin{array}{ccccc}
2 & 0 & 0 & 0 & 0 \\
0 & 0 & 0 & 0 \\
0 & 0 & 0 & 0 \\
0 & 0 & 0 & 0
\end{array}\right)
$$

generate $U=I_{4}$, while only $H^{\prime}$ can be used to approximate some non-identity evolutions. Thus one should give either a prescription for the choice of the generating Hamiltonian or a proof that different choices generically have the same power, but neither was provided in 8 .

2. The argument makes use of the fact that any gate $A$ given by (4) is universal. However, such gates are only universal with ancillae (because $|00\rangle$ is a fixed-point of both $A$ and $T A T$, so composing them cannot approximate any $U \in \mathrm{U}(4)$ that does not fix $|00\rangle)$, yet the final result claims universality without the need for ancillae.

3. The argument proceeds by considering a Hamiltonian $H_{1}$ that generates the gate $A$. The authors claim that $H_{1}$ is universal due to the linear independence of the following 16 nested commutators of $H_{1}$ and $T H_{1} T$ :

$$
\begin{aligned}
H_{1}, & \\
H_{2} & :=T H_{1} T, \\
H_{j} & :=i\left[H_{1}, H_{j-1}\right], \quad j \in\{3, \ldots, 14\}, \\
H_{15} & :=i\left[H_{2}, H_{3}\right], \\
H_{16} & :=i\left[H_{2}, H_{5}\right],
\end{aligned}
$$

However, as in item 1, the claim may or may not hold depending on the choice of the Hamiltonian $H_{1}$ generating $A$. In fact, the most natural choice,

$$
H_{1}:=\left(\begin{array}{cccc}
0 & 0 & 0 & 0 \\
0 & 0 & 0 & 0 \\
0 & 0 & \beta & -\theta e^{-i \phi} \\
0 & 0 & -\theta e^{i \phi} & \beta
\end{array}\right),
$$

does not generate $\mathfrak{u}(4)$ since the entire Lie algebra fixes $|00\rangle$. However, there are other choices of $H_{1}$ for which $H_{1}, \ldots, H_{16}$ are linearly independent. For example, if one chooses $H_{1}$ to act diagonally in a random basis on the degenerate 1-eigenspace of $A$, with eigenvalues $2 \pi$ and $4 \pi$, then $H_{1}, \ldots, H_{16}$ are found to be linearly independent in a numerical experiment.

For any explicit Hamiltonian $H$, it is simple to generate the 16 matrices according to (6) and their linear dependence is easily checked. If these 16 matrices are linearly independent, then we say that (6) certifies the universality of $H$.

4. To show that almost any unitary gate is universal, non-universal gates are argued to lie in a submanifold of $\mathrm{U}(4)$ of at most 15 dimensions. The argument begins by considering a one-parameter family of Hamiltonians $H(k)=H+k(\tilde{H}-H)$ where $k \in \mathbb{R}, H$ is arbitrary, and $\tilde{H}$ is a fixed Hamiltonian whose universality is certified by (6). Then, unless $k$ is a root of a certain polynomial of finite degree, (6) also certifies the universality of $H(k)$. This argument is claimed to extend to a 16-dimensional neighborhood of $H$ (which could be parametrized as $H\left(k_{1}, \ldots, k_{16}\right)=H+k_{1}\left(\tilde{H}^{(1)}-H\right)+\cdots+$ $\left.k_{16}\left(\tilde{H}^{(16)}-H\right)\right)$. However, the explicit analysis of the relevant multivariate polynomial is omitted. Furthermore, the argument requires that $(6)$ certifies the universality of each of $\tilde{H}^{(1)}, \ldots, \tilde{H}^{(16)}$, but this is not demonstrated, and it is unclear to us whether it actually holds for some choice of $H_{1}$.

Reference [8] also conjectures that a 2-qubit unitary gate is non-universal if and only if it

1. permutes states of some orthonormal basis or

2. is a tensor product of single-qubit unitary gates. 
We note that a unitary gate $U$ satisfying item 1 need not be non-universal, because $U$ and TUT may not permute the same basis. We presume that the authors of $[8]$ intended to require that both $U$ and TUT permute states of the same orthonormal basis.

In Theorem 2 of this paper, we disprove the above conjecture and give a complete characterization of the set of non-universal 2-qubit Hamiltonians, thereby resolving a variant of the above question.

\subsection{Proving universality}

The first step in our quest for a simple closed-form characterization of universal Hamiltonians is a characterization of universality in terms of Lie algebras, just as in [8, 14].

Definition 6. We write $\mathcal{L}\left(H_{1}, \ldots, H_{k}\right)$ to denote the Lie algebra generated by Hamiltonians $H_{1}, \ldots, H_{k}$. It is defined inductively by the following three rules:

1. $H_{1}, \ldots, H_{k} \in \mathcal{L}\left(H_{1}, \ldots, H_{k}\right)$,

2. if $A, B \in \mathcal{L}\left(H_{1}, \ldots, H_{k}\right)$ then $\alpha A+\beta B \in \mathcal{L}\left(H_{1}, \ldots, H_{k}\right)$ for all $\alpha, \beta \in \mathbb{R}$, and

3. if $A, B \in \mathcal{L}\left(H_{1}, \ldots, H_{k}\right)$ then $i[A, B]:=i(A B-B A) \in \mathcal{L}\left(H_{1}, \ldots, H_{k}\right)$.

The set of evolutions that can be simulated using a set of Hamiltonians is given by the following lemma:

Lemma 1. Assume that we can evolve according to Hamiltonians $H_{1}, \ldots, H_{k}$ for any desired amount of time. Then we can simulate the unitary $U$ if and only if

$$
U \in \operatorname{cl}\left\{e^{-i L}: L \in \mathcal{L}\left(H_{1}, \ldots, H_{k}\right)\right\},
$$

where "cl" denotes the closure of a set 1

One can easily prove the above lemma using the Lie product formula, the analogous formula for $e^{[A, B]}$, and the Campbell-Baker-Hausdorff formula [11].

Now we can obtain a simpler and more practical sufficient condition for $n$-universality than the original one from Definition 3 ,

Corollary 1. Let $m \leq n$. Then an $m$-qubit Hamiltonian $H$ is $n$-universal if

$$
\mathcal{L}\left(\left\{P\left(H \otimes I^{\otimes n-m}\right) P^{\dagger}: P \in \mathcal{S}_{n}\right\}\right)=\mathfrak{u}\left(2^{n}\right),
$$

where $\mathcal{S}_{n}$ is the group of matrices that permute $n$ qubits and $\mathfrak{u}\left(2^{n}\right)$ is the set of all $2^{n} \times 2^{n}$ Hermitian matrices. In particular, a 2-qubit Hamiltonian $H$ is 2-universal if $\mathcal{L}(H, T H T)=\mathfrak{u}(4)$, where $\mathfrak{u}(4)$ is the set of all $4 \times 4$ Hermitian matrices.

Now we proceed to show that if a Hamiltonian $H$ is $n$-universal then it is also $n^{\prime}$-universal for all $n^{\prime} \geq n$. Note that this is not completely trivial, since the added qubits are not ancillary, i.e., we have to be able to simulate any unitary on all of the qubits.

Lemma 2. If a Hamiltonian $H$ is $n$-universal for some $n \geq 2$, then it is also $n^{\prime}$-universal for all $n^{\prime} \geq n$. In particular, a 2-universal 2-qubit Hamiltonian $H$ is also $n$-universal for all integers $n \geq 2$.

Proof. Since $H$ is $n$-universal for some $n \geq 2$, it can be used to simulate all unitary transformations in $\mathrm{U}\left(2^{n^{\prime}}\right)$ that act non-trivially on no more than two qubits. But any unitary gate on $n^{\prime}$ qubits can be decomposed into gates that act non-trivially only on one or two qubits without the need for ancillae 2, 16, so $H$ is $n^{\prime}$-universal.

\footnotetext{
${ }^{1}$ This is false without the closure. For example, consider $H=\left(\begin{array}{cc}1 & 0 \\ 0 & \sqrt{2}\end{array}\right)$. We can use $H$ to simulate any diagonal $2 \times 2$ unitary but there are diagonal unitary matrices such as $\left(\begin{array}{cc}1 & 0 \\ 0 & -1\end{array}\right)$ that are not of the form $e^{-i H t}$ for $t \in \mathbb{R}$.
} 


\section{Characterization of 2-universal Hamiltonians}

In this section we classify the set of 2-qubit Hamiltonians that are not 2-universal. Since we only consider 2-universality, we simply say that a Hamiltonian is universal (instead of "2-universal") or non-universal (instead of "not 2-universal") for the remainder of this section.

Our analysis relies on an equivalence relation that partitions the set of all 2-qubit Hamiltonians into equivalence classes, each containing only universal or non-universal Hamiltonians (but not both). First we identify three families of non-universal Hamiltonians and extend each family to the union of the equivalence classes containing its family members. Then we show that each subset contains a special element whose universality (or non-universality) can be succinctly characterized. This allows us to show universality of any Hamiltonian not belonging to any of the three generalized non-universal families.

\subsection{The $T$ gate and the $T$-basis}

The gate $T$ that swaps two qubits is of central importance since it is the only non-trivial permutation of two qubits. Recall that its matrix representation in the computational basis is

$$
T:=\left(\begin{array}{cccc}
1 & 0 & 0 & 0 \\
0 & 0 & 1 & 0 \\
0 & 1 & 0 & 0 \\
0 & 0 & 0 & 1
\end{array}\right) .
$$

It has two eigenspaces, namely

$$
E_{-}:=\operatorname{span}_{\mathbb{C}}\{|01\rangle-|10\rangle\} \quad \text { and } \quad E_{+}:=\operatorname{span}_{\mathbb{C}}\{|00\rangle,|01\rangle+|10\rangle,|11\rangle\},
$$

where $E_{-}$corresponds to the eigenvalue -1 and $E_{+}$to the eigenvalue +1 . The normalized vector

$$
|s\rangle:=\frac{|01\rangle-|10\rangle}{\sqrt{2}}
$$

that spans $E_{-}$is called the singlet state.

We prove the following basic facts about the $T$ gate in Appendix B

Fact 1. The singlet $|s\rangle$ is an eigenvector of a normal matrix $N \in \mathrm{M}_{4}(\mathbb{C})$ if and only if $[N, T]=0$.

Fact 2. A normal matrix $N \in \mathrm{M}_{4}(\mathbb{C})$ has a common eigenvector with the $T$ gate if and only if it has an eigenvector orthogonal to $|s\rangle$.

Fact 3. Suppose $U \in \mathrm{U}(4)$ and $[U, T]=0$. Then the singlet state $|s\rangle$ is an eigenvector of both $U$ and $U^{\dagger}$.

We will use both the computational basis and one in which $T$ is diagonal, with the singlet state as the first basis vector. For definiteness, we choose

$$
U_{T}:=\frac{1}{\sqrt{2}}\left(\begin{array}{cccc}
0 & 1 & -1 & 0 \\
0 & 1 & 1 & 0 \\
1 & 0 & 0 & 1 \\
1 & 0 & 0 & -1
\end{array}\right)
$$

to implement the basis change. We call the resulting basis the $T$-basis. The $T$ gate and the singlet state become $\tilde{T}:=U_{T} T U_{T}^{\dagger}$ and $|\tilde{s}\rangle:=U_{T}|s\rangle$ given by

$$
\tilde{T}=\left(\begin{array}{cccc}
-1 & 0 & 0 & 0 \\
0 & 1 & 0 & 0 \\
0 & 0 & 1 & 0 \\
0 & 0 & 0 & 1
\end{array}\right) \quad \text { and } \quad|\tilde{s}\rangle=\left(\begin{array}{l}
1 \\
0 \\
0 \\
0
\end{array}\right)
$$




\subsection{Three simple families of non-universal Hamiltonians}

Three families of non-universal Hamiltonians are easily identified.

Fact 4. A two-qubit Hamiltonian $H$ is non-universal if any of the following conditions holds:

1. $H$ is a local Hamiltonian, i.e., $H=H_{1} \otimes I+I \otimes H_{2}$, for some 1-qubit Hamiltonians $H_{1}, H_{2}$,

2. $H$ shares an eigenvector with the $T$ gate, or

3. $\operatorname{Tr}(H)=0$.

In Section 3.4 we extend these families to larger sets of non-universal Hamiltonians, so the above do not literally exhaust the set of non-universal Hamiltonians. However, we prove in Section 3.5 that the extended families contain all non-universal Hamiltonians, so these three families do capture the essence of what makes a Hamiltonian non-universal.

\section{$3.3 \quad T$-similarity}

The following equivalence relation between Hamiltonians is central to our analysis:

Definition 7. We say that matrices $A$ and $B$ are $T$-similar if there exists a unitary matrix $P$ such that $B=P A P^{\dagger}$ and $[P, T]=0$.

Conjugation by $P$ preserves universality, i.e., it maps universal 2-qubit Hamiltonians to universal Hamiltonians and non-universal 2-qubit Hamiltonians to non-universal Hamiltonians. In particular:

Theorem 1. Let $A, B$ be $T$-similar 2-qubit Hamiltonians. Then $A$ is universal if and only if $B$ is.

Proof. Assume 2-qubit Hamiltonians $A$ and $B$ are $T$-similar. Then there is some $P \in \mathrm{U}(4)$ such that $B=P A P^{\dagger}$ and $[P, T]=0$. Suppose $A$ is universal. We want to show that $B$ is also universal. We have to show that using $B$ we can simulate any $U \in \mathrm{U}(4)$ with any desired precision $\varepsilon>0$. Since $A$ is universal, we can simulate $P^{\dagger} U P \in \mathrm{U}(4)$ with precision $\varepsilon$, i.e., there exists $n \in \mathbb{N}$ and $t_{1}, \ldots, t_{n} \geq 0$ such that

$$
\left\|P^{\dagger} U P-e^{-i A t_{1}} e^{-i T A T t_{2}} e^{-i A t_{3}} \ldots e^{-i T A T t_{n}}\right\|_{\infty}<\varepsilon
$$

Since $T P=P T, B=P A P^{\dagger}$ and $e^{V M V^{\dagger}}=V e^{M} V^{\dagger}$ for all unitary $V$ and all matrices $M$, we have

$$
e^{-i B t_{1}} e^{-i T B T t_{2}} e^{-i B t_{3}} \ldots e^{-i T B T t_{n}}=P e^{-i A t_{1}} e^{-i T A T t_{2}} e^{-i A t_{3}} \ldots e^{-i T A T t_{n}} P^{\dagger} .
$$

Combining (15) with 16$)$ and noting that the spectral norm is invariant under unitary conjugation gives

$$
\left\|U-e^{-i B t_{1}} e^{-i T B T t_{2}} e^{-i B t_{3}} \ldots e^{-i T B T t_{n}}\right\|_{\infty}<\varepsilon .
$$

Hence $e^{-i B t_{1}} e^{-i T B T t_{2}} e^{-i B t_{3}} \ldots e^{-i T B T t_{n}}$ is the desired simulation of $U$ with precision $\varepsilon$. We conclude that $B$ is universal.

Thus $T$-similarity partitions the set of all 2-qubit Hamiltonians into equivalence classes, each containing only universal or non-universal Hamiltonians.

\subsection{Three extended families of non-universal Hamiltonians}

In view of Theorem 1 1 , each family of non-universal Hamiltonians in Fact 4 can be extended to include Hamiltonians that are $T$-similar to its elements. We now analyze each of these three extended families.

1. T-similarity transformations do not preserve the set of local Hamiltonians. For example, when

$$
H:=\left(\begin{array}{ll}
1 & 0 \\
0 & 0
\end{array}\right) \otimes I+I \otimes\left(\begin{array}{cc}
1 & 0 \\
0 & 0
\end{array}\right) \quad \text { and } \quad P:=\left(\begin{array}{cccc}
\frac{1}{\sqrt{2}} & 0 & 0 & \frac{1}{\sqrt{2}} \\
0 & 0 & 1 & 0 \\
0 & 1 & 0 & 0 \\
\frac{1}{\sqrt{2}} & 0 & 0 & -\frac{1}{\sqrt{2}}
\end{array}\right),
$$

$H$ is local and $P$ commutes with $T$, but $P H P^{\dagger}=I \otimes I+\frac{1}{2}\left[\left(\begin{array}{ll}0 & 1 \\ 1 & 0\end{array}\right) \otimes\left(\begin{array}{ll}0 & 1 \\ 1 & 0\end{array}\right)-\left(\begin{array}{cc}0 & -i \\ i & 0\end{array}\right) \otimes\left(\begin{array}{cc}0 & -i \\ i & 0\end{array}\right)\right]$ which is non-local. Thus the extended family is strictly larger. 
2. $T$-similarity transformations preserve the property of sharing an eigenvector with the $T$ gate:

Lemma 3. The set of two-qubit Hamiltonians sharing an eigenvector with the $T$ gate is closed under conjugation by unitary transformations that commute with $T$.

Proof. Let $U$ satisfy $[U, T]=0$ and let $|v\rangle$ be the eigenvector shared by $H$ and the $T$ gate, i.e., $H|v\rangle=\lambda_{H}|v\rangle$ and $T|v\rangle=\lambda_{T}|v\rangle$ for some $\lambda_{H}, \lambda_{T}$. We claim that $U|v\rangle$ is an eigenvector shared by the $T$ gate and $U H U^{\dagger}$. First, note that $U H U^{\dagger}(U|v\rangle)=U H|v\rangle=\lambda_{H} U|v\rangle$. We also have $T(U|v\rangle)=$ $U T|v\rangle=\lambda_{T} U|v\rangle$. Thus $U|v\rangle$ is an eigenvector shared by the $T$ gate and $U H U^{\dagger}$.

Therefore, the extension does not add more non-universal Hamiltonians to this family.

3. The set of traceless Hamiltonians is preserved by $T$-similarity transformations.

Using the above, we generalize Fact 4 to the following:

Lemma 4. A two-qubit Hamiltonian $H$ is non-universal if any of the following conditions holds:

1. $H$ is $T$-similar to a local Hamiltonian,

2. $H$ shares an eigenvector with the $T$ gate, or

3. $\operatorname{Tr}(H)=0$.

Another easily recognized family of non-universal Hamiltonians is the set of generators of orthogonal transformations. However, this set can be shown to be contained in the first family of the above lemma [15]. Similarly, Hamiltonians with degenerate eigenvalues can be shown to be non-universal, since they always share an eigenvector with the $T$ gate (see Fact 5 in Appendix B).

It is straightforward to check whether a given Hamiltonian belongs to the last two families of non-universal Hamiltonians in Lemma 4. The following lemma (proved in Appendix C) gives an efficient method to check whether a given Hamiltonian with non-degenerate eigenvalues is $T$-similar to a local Hamiltonian.

Lemma 5. A 2-qubit Hamiltonian is $T$-similar to a local Hamiltonian if and only if it has an orthonormal basis of eigenvectors $\left|v_{1}\right\rangle,\left|v_{2}\right\rangle,\left|v_{3}\right\rangle,\left|v_{4}\right\rangle$ corresponding to the eigenvalues $\lambda_{1}, \lambda_{2}, \lambda_{3}, \lambda_{4}$ so that

1. $\left|\left\langle v_{1} \mid s\right\rangle\right|=\left|\left\langle v_{2} \mid s\right\rangle\right|$ and $\left|\left\langle v_{3} \mid s\right\rangle\right|=\left|\left\langle v_{4} \mid s\right\rangle\right|$, and

2. $\lambda_{1}+\lambda_{2}=\lambda_{3}+\lambda_{4}$,

where $|s\rangle$ is the singlet state defined in equation $(12)$.

\subsection{The three extended families of non-universal Hamiltonians are exhaustive}

In this section we show that the list of non-universal families of Hamiltonians in Lemma 4 is in fact complete. This is done by analyzing a special member of each $T$-similarity equivalence class.

\subsubsection{Tridiagonal form}

We now introduce a normal form for 2-qubit Hamiltonians.

Definition 8. We say that a 2-qubit Hamiltonian is in tridiagonal form if it is of the form

$$
\left(\begin{array}{llll}
a & b & 0 & 0 \\
b & c & d & 0 \\
0 & d & e & f \\
0 & 0 & f & g
\end{array}\right),
$$

where $a, b, c, d, e, f, g \in \mathbb{R}$ and $b, d, f \geq 0$. If either of $b, d$ is 0 , we additionally require that

- if $b=0$, then $d=f=0$ and $c \geq e \geq g$, and 
- if $d=0$, then $f=0$ and $e \geq g$.

Note that a tridiagonal Hamiltonian is of one of the following types:

$$
\begin{aligned}
& \left(\begin{array}{cccc}
* & + & 0 & 0 \\
+ & * & + & 0 \\
0 & + & * & + \\
0 & 0 & + & *
\end{array}\right) \quad\left(\begin{array}{cccc}
* & + & 0 & 0 \\
+ & * & + & 0 \\
0 & + & * & 0 \\
0 & 0 & 0 & *
\end{array}\right) \quad\left(\begin{array}{cccc}
* & + & 0 & 0 \\
+ & * & 0 & 0 \\
0 & 0 & *_{1} & 0 \\
0 & 0 & 0 & *_{2}
\end{array}\right) \quad\left(\begin{array}{cccc}
* & 0 & 0 & 0 \\
0 & *_{1} & 0 & 0 \\
0 & 0 & *_{2} & 0 \\
0 & 0 & 0 & *_{3}
\end{array}\right) \\
& \begin{array}{llll}
\text { Type } 1 & \text { Type } 2 & \text { Type } 3 & \text { Type } 4
\end{array}
\end{aligned}
$$

where $*_{1} \geq *_{2} \geq *_{3}$ and "+" stands for a positive entry and " $*$ " for any real entry.

When given a 2-qubit Hamiltonian in tridiagonal form, we will often use the letters $a, b, c, d, e, f, g$ to refer to its entries as indicated in equation 19 .

Definition 9. For any 2-qubit Hamiltonian $H$, we say that $\Xi$ is a tridiagonal form of $H$ if $H$ and $\Xi$ are $T$-similar and $\Xi$ is tridiagonal in the $T$-basis. (We will use $\tilde{\Xi}:=U_{T} \Xi U_{T}^{\dagger}$ to denote $\Xi$ in the $T$-basis.)

It follows from the definition that $T$-similar 2-qubit Hamiltonians share the same tridiagonal forms (if they exist). We now show that for every 2-qubit Hamiltonian a tridiagonal form indeed exists and is in fact unique. Thus, each equivalence class is uniquely characterized by the tridiagonal form of its Hamiltonians.

Lemma 6. Every 2-qubit Hamiltonian $H$ has a unique tridiagonal form $\Xi$.

Proof. Since $T$-similarity is basis-independent, we prove the lemma in the $T$-basis. In other words, we prove that $\tilde{H}:=U_{T} H U_{T}^{\dagger}$ is $T$-similar to a unique tridiagonal matrix. Note that in the $T$-basis, $T$-similar matrices are related by conjugation by some unitary $V \in \mathrm{U}(1) \oplus \mathrm{U}(3)$.

Let the first column of $\tilde{H}$ be $\left(h_{1}, h_{2}, h_{3}, h_{4}\right)^{\mathrm{T}}$, where $\left\|\left(h_{2}, h_{3}, h_{4}\right)^{\mathrm{T}}\right\|=b \geq 0$. Then we can find $P_{1} \in$ $I_{1} \oplus \mathrm{U}(3)$ such that the first column of $\tilde{H}_{1}:=P_{1} \tilde{H} P_{1}^{\dagger}$ is $\left(h_{1}, b, 0,0\right)^{\top}$. Now let the second column of $\tilde{H}_{1}$ be $\left(h_{1}^{\prime}, h_{2}^{\prime}, h_{3}^{\prime}, h_{4}^{\prime}\right)^{\mathrm{T}}$, where $\left\|\left(h_{3}^{\prime}, h_{4}^{\prime}\right)^{\mathrm{T}}\right\|=d \geq 0$, and choose $P_{2} \in I_{2} \oplus \mathrm{U}(2)$ such that the second column of $\tilde{H}_{2}:=P_{2} \tilde{H}_{1} P_{2}^{\dagger}$ is $\left(h_{1}^{\prime}, h_{2}^{\prime}, d, 0\right)^{\top}$. Note that the first column of $\tilde{H}_{2}$ remains the same as for $\tilde{H}_{1}$. Finally, we can find $P_{3} \in I_{3} \oplus \mathrm{U}(1)$ such that the last entry $f$ of the third column of $\tilde{H}_{3}:=P_{3} \tilde{H}_{2} P_{3}^{\dagger}$ is real and non-negative. Since $\tilde{H}_{3}$ is Hermitian, its diagonal entries are real and it has the form (19). If neither $b$ nor $d$ is zero, we are done. If $b=0$, we diagonalize the lower right $3 \times 3$ block of $\tilde{H}_{3}$ by conjugating with unitary transformations of the form $1 \oplus \mathrm{U}(3)$. Similarly, if $d=0$ we diagonalize the lower right $2 \times 2$ block. Thus we obtain a tridiagonal form of $H$.

Now we show that $\Xi$ is unique. If $\Xi_{1}$ and $\Xi_{2}$ are both tridiagonal forms of $H$, then in the $T$-basis $\tilde{\Xi}_{1}$ and $\tilde{\Xi}_{2}$ are related by conjugation by some $V \in \mathrm{U}(1) \oplus \mathrm{U}(3)$. We first consider $\tilde{\Xi}_{1}$ of type 1 . Since the first column of $\tilde{\Xi}_{2}$ has to be of the form $(a, b, 0,0)^{\top}$ for some $a, b \in \mathbb{R}, b>0, V$ has to be of the form $e^{i \varphi} I_{2} \oplus \mathrm{U}(2)$ for some $\varphi \in \mathbb{R}$. Similarly, by considering the second and third columns of $\tilde{\Xi}_{2}$, we conclude that $V=e^{i \varphi} I_{4}$. Thus, we have $\tilde{\Xi}_{2}=\left(e^{i \varphi} I_{4}\right) \tilde{\Xi}_{1}\left(e^{-i \varphi} I_{4}\right)=\tilde{\Xi}_{1}$. If $\tilde{\Xi}_{1}$ is of type 2,3 , or 4 , similar reasoning can be applied; in each case, the form of $V$ is constrained so that $\tilde{\Xi}_{1}=\tilde{\Xi}_{2}$.

\subsubsection{Tridiagonal forms of non-universal Hamiltonians}

In this section we give a simple characterization of the three families of non-universal Hamiltonians listed in Lemma 4 in terms of their tridiagonal forms.

Lemma 7. Let $H$ be a 2-qubit Hamiltonian and let $\Xi$ be its tridiagonal form, with $\tilde{\Xi}$ given by equation 19 . Then $H$ has a common eigenvector with the $T$ gate if and only if $b d f=0$.

Proof. By Fact 2, $H$ has a common eigenvector with $T$ if and only if $H$ has an eigenvector orthogonal to the singlet $|s\rangle$. By definition of the tridiagonal form, there is a unitary conjugating $H$ to $\tilde{\Xi}, T$ to $\tilde{T}$, and taking $|s\rangle$ to $|\tilde{s}\rangle$. Thus it suffices to show that $\tilde{\Xi}$ has an eigenvector orthogonal to $|\tilde{s}\rangle$ if and only if $b d f=0$.

If $b d f=0$, then $\tilde{\Xi}$ has an invariant subspace orthogonal to $|\tilde{s}\rangle$. This subspace has dimension 3,2 , or 1 if $b=0, d=0$, or $f=0$, respectively. In any case, it contains at least one eigenvector, so $\tilde{\Xi}$ has an eigenvector orthogonal to $|\tilde{s}\rangle$. 
If $\tilde{\Xi}$ has an eigenvector $|\tilde{v}\rangle$ that is orthogonal to $|\tilde{s}\rangle$, then $|\tilde{v}\rangle=\left(0, v_{2}, v_{3}, v_{4}\right)^{\top}$ for some $v_{2}, v_{3}, v_{4} \in \mathbb{C}$, not all zero. Since $\tilde{\Xi}$ is Hermitian, we have $\tilde{\Xi}|\tilde{v}\rangle=r|\tilde{v}\rangle$ for some $r \in \mathbb{R}$, or equivalently,

$$
\left(\begin{array}{c}
b v_{2} \\
c v_{2}+d v_{3} \\
d v_{2}+e v_{3}+f v_{4} \\
f v_{3}+g v_{4}
\end{array}\right)=\left(\begin{array}{c}
0 \\
r v_{2} \\
r v_{3} \\
r v_{4}
\end{array}\right) .
$$

From the first entry, $b v_{2}=0$ so that $b=0$ or $v_{2}=0$. If $b \neq 0$, then $v_{2}=0$ and from the second entry, $d v_{3}=0$, so either $d=0$ or $v_{3}=0$. If $d \neq 0$, then $v_{3}=0$ and from the third entry, $f v_{4}=0$ and so, if $f \neq 0$, $v_{4}=0$ which contradicts $|\tilde{v}\rangle \neq 0$. Thus, $b d f=0$.

Lemma 8. Let $H$ be a 2-qubit Hamiltonian not sharing an eigenvector with $T$ and let $\Xi$ be its tridiagonal form. Then $H$ is $T$-similar to a local Hamiltonian if and only if $a=c=e=g$ for $\tilde{\Xi}$ as given in equation [19].

Proof. Assume $a=c=e=g$. $H$ is $T$-similar to a local Hamiltonian if and only if $\Xi$ is, so we can apply Lemma 5 to $\Xi$. A straightforward calculation gives eigenvectors $\left|v_{i, j}\right\rangle$ of $\tilde{\Xi}$ for $i, j \in\{0,1\}$, with eigenvalues

$$
\lambda_{i, j}=a+(-1)^{i} \sqrt{\frac{b^{2}+d^{2}+f^{2}+(-1)^{j} z}{2}} \quad \text { where } z:=\sqrt{b^{4}+d^{4}+f^{4}+2\left(b^{2} d^{2}+d^{2} f^{2}-b^{2} f^{2}\right)} .
$$

The overlaps of these eigenvectors with the singlet state are

$$
\left|\left\langle v_{i, j} \mid \tilde{s}\right\rangle\right|=\sqrt{\frac{z+(-1)^{j}\left(b^{2}-d^{2}-f^{2}\right)}{4 z}} .
$$

For each $j \in\{0,1\}, \lambda_{0, j}+\lambda_{1, j}=2 a$ and $\left|\left\langle v_{0, j} \mid \tilde{s}\right\rangle\right|=\left|\left\langle v_{1, j} \mid \tilde{s}\right\rangle\right|$, so both conditions in Lemma 5 are satisfied. Hence $H$ is $T$-similar to a local Hamiltonian.

Now assume that $H$ is $T$-similar to a local Hamiltonian. We prove that $\tilde{\Xi}$ has $a=c=e=g$ by explicitly computing it. We do this in two steps: first we show that $H$ is $T$-similar to some $H^{\prime}$ of the form

$$
H^{\prime}=\alpha I_{4}+\left(x_{1} X+z_{1} Z\right) \otimes I+I \otimes\left(z_{2} Z\right)
$$

for some $\alpha, x_{1}, z_{1}, z_{2} \in \mathbb{R}$ (where $I:=\left(\begin{array}{ll}1 & 0 \\ 0 & 1\end{array}\right), X:=\left(\begin{array}{cc}0 & 1 \\ 1 & 0\end{array}\right), Y:=\left(\begin{array}{cc}0 & -i \\ i & 0\end{array}\right), Z:=\left(\begin{array}{cc}1 & 0 \\ 0 & -1\end{array}\right)$ are Pauli matrices), and then we find the common tridiagonal form of $H$ and $H^{\prime}$.

Step 1. Consider conjugating $H$ by $U \otimes U$ where $U \in \mathrm{SU}(2)$ (clearly, $[U \otimes U, T]=0$ ). It suffices to consider a local Hamiltonian

$$
H=H_{1} \otimes I+I \otimes H_{2}
$$

for some 1-qubit Hamiltonians $H_{1}$ and $H_{2}$. Pick $V \in \mathrm{SU}(2)$ such that $V H_{2} V^{\dagger}$ is diagonal. Then pick a diagonal matrix $D \in \mathrm{SU}(2)$ such that $D V H_{1} V^{\dagger} D^{\dagger}$ is a real matrix. Note that $D V H_{2} V^{\dagger} D^{\dagger}$ is still diagonal. Therefore, $U H U^{\dagger}$ is of the form (23), where $U:=(D V) \otimes(D V)$.

Step 2. Recall that $H^{\prime}$ in the $T$-basis is given by $\tilde{H}^{\prime}:=U_{T} H^{\prime} U_{T}^{\dagger}$. Using equation (23), we get

$$
\tilde{H}^{\prime}=\alpha(I \otimes I)+x_{1}(Y \otimes Y)+z_{1}(I \otimes X)-z_{2}(Z \otimes X)=\left(\begin{array}{cccc}
\alpha & z_{1}-z_{2} & 0 & -x_{1} \\
z_{1}-z_{2} & \alpha & x_{1} & 0 \\
0 & x_{1} & \alpha & z_{1}+z_{2} \\
-x_{1} & 0 & z_{1}+z_{2} & \alpha
\end{array}\right) .
$$

Note that $x_{1} \neq 0$, or else, by Lemma 7 , we contradict the fact that $H$ does not share an eigenvector with $T$. We apply one more $T$-similarity transformation to bring $\tilde{H}^{\prime}$ into tridiagonal form. Let $l:=\sqrt{x_{1}^{2}+\left(z_{1}-z_{2}\right)^{2}}>0$ and

$$
Q:=\left(\begin{array}{cccc}
1 & 0 & 0 & 0 \\
0 & \frac{z_{1}-z_{2}}{l} & 0 & \frac{x_{1}}{l} \\
0 & 0 & 1 & 0 \\
0 & \frac{-x_{1}}{l} & 0 & \frac{z_{1}-z_{2}}{l}
\end{array}\right)
$$


which is in $I_{1} \oplus \mathrm{U}(3)$. Then

$$
Q^{\dagger} \tilde{H}^{\prime} Q=\left(\begin{array}{cccc}
\alpha & l & 0 & 0 \\
l & \alpha & \frac{-2 x_{1} z_{2}}{l} & 0 \\
0 & \frac{-2 x_{1} z_{2}}{l} & \alpha & \frac{x_{1}^{2}+z_{1}^{2}-z_{2}^{2}}{l} \\
0 & 0 & \frac{x_{1}^{2}+z_{1}^{2}-z_{2}^{2}}{l} & \alpha
\end{array}\right)
$$

Since $H$ does not share an eigenvector with $T$, the $(2,1),(3,2),(4,3)$ entries of $Q^{\dagger} \tilde{H}^{\prime} Q$ are all nonzero. Their signs can be made positive by conjugation with a diagonal matrix with diagonal entries \pm 1 , preserving the diagonal elements (note that this is a $T$-similarity transformation). This tridiagonal form of $H^{\prime}$ and $H$ has equal diagonal entries as claimed.

Using Lemmas 7 and 8 , we can restate Lemma 4 in terms of the tridiagonal form:

Corollary 2. Let $H$ be a 2-qubit Hamiltonian with tridiagonal form $\Xi$, with $\tilde{\Xi}$ given by 19 . Then $H$ is non-universal if

1. $\tilde{\Xi}$ has $b d f=0$,

2. $\tilde{\Xi}$ has $a=c=e=g$, or

3. $\tilde{\Xi}$ has $a+c+e+g=0$.

\subsubsection{Universality certificate for tridiagonal Hamiltonians}

Given a Hamiltonian $H$ that does not satisfy any of the conditions of Corollary 2 we provide a list of 16 linearly independent linear combinations of nested commutators of $\tilde{\Xi}$ and $\tilde{T} \Xi T$. This shows that $\mathcal{L}(H, T H T)=\mathcal{L}(\tilde{\Xi}, \tilde{T} \tilde{\Xi} \tilde{T})=\mathfrak{u}(4)$. Hence it follows from Corollary 1 that $H$ is universal.

Let $E_{k, l}:=|k\rangle\langle l|$ and define a basis for $\mathfrak{s u}(4)$ (i.e., for traceless $4 \times 4$ Hermitian matrices) as follows:

$$
\begin{array}{rlrl}
X_{k, l} & :=E_{k, l}+E_{l, k}, & (1 \leq k<l \leq 4) \\
Y_{k, l} & :=-i E_{k, l}+i E_{l, k}, & (1 \leq k<l \leq 4) \\
Z_{k}:=E_{k, k}-E_{k+1, k+1} . & (1 \leq k \leq 3)
\end{array}
$$

These 15 matrices together with any Hermitian matrix with non-zero trace form a basis for $\mathfrak{u}(4)$. We now obtain these basis vectors as nested commutators of $\tilde{\Xi}$ and $\tilde{T} \tilde{\Xi} \tilde{T}$.

By violation of the first condition in Corollary $2, b d f \neq 0$. Thus we can generate $A:=\frac{1}{2 b} i[\tilde{\Xi}, \tilde{T} \tilde{\Xi} \tilde{T}]$ and

$$
\begin{aligned}
X_{1,2} & =\frac{1}{2 b}(\tilde{\Xi}-\tilde{T} \tilde{\Xi} \tilde{T}), \\
Y_{1,3} & =\frac{1}{3 d}\left(i\left[i\left[X_{1,2}, A\right], X_{1,2}\right]-4 A\right), \\
X_{2,3} & =i\left[X_{1,2}, Y_{1,3}\right] .
\end{aligned}
$$

Next, we can generate $B:=\frac{1}{2}(\tilde{\Xi}+\tilde{T} \tilde{\Xi} \tilde{T})$. To obtain $Y_{1,2}$ we consider three cases:

$$
Y_{1,2}= \begin{cases}\frac{1}{a-c}\left(d Y_{1,3}+A\right) & \text { if } a \neq c, \\ \frac{1}{c-e} i\left[Y_{1,3}, i\left[B, X_{2,3}\right]\right] & \text { if } c \neq e, \\ \frac{1}{a-g} \frac{1}{f^{2}} i\left[i\left[X_{2,3}, B\right], i\left[B, i\left[Y_{1,3}, B\right]\right]\right] & \text { otherwise }(a=c=e \neq g) .\end{cases}
$$

One of these cases has to hold since the second condition in Corollary 2 is violated. We next obtain

$$
\begin{aligned}
& X_{1,3}=i\left[Y_{1,2}, X_{2,3}\right], \\
& X_{1,4}=\frac{1}{f}\left((c-e) X_{1,3}+i\left[A, X_{2,3}\right]+i\left[Y_{1,3}, B\right]\right) .
\end{aligned}
$$


We obtain the remaining basis elements as follows:

$$
\begin{aligned}
& X_{2,4}=i\left[X_{1,4}, Y_{1,2}\right], \quad X_{3,4}=i\left[X_{1,4}, Y_{1,3}\right], \quad Y_{1,4}=i\left[X_{2,4}, X_{1,2}\right], \\
& Y_{2,3}=i\left[X_{1,3}, X_{1,2}\right], \quad Y_{2,4}=i\left[X_{1,4}, X_{1,2}\right], \quad Y_{3,4}=i\left[X_{1,4}, X_{1,3}\right] \text {, } \\
& Z_{1}=\frac{1}{2} i\left[Y_{1,2}, X_{1,2}\right], \quad Z_{2}=\frac{1}{2} i\left[Y_{2,3}, X_{2,3}\right], \quad Z_{3}=\frac{1}{2} i\left[Y_{3,4}, X_{3,4}\right] .
\end{aligned}
$$

At this point we can generate $\mathfrak{s u}(4)$. If the third condition in Corollary 2 does not hold, then $\operatorname{Tr}(\tilde{\Xi}) \neq 0$, so adding $\tilde{\Xi}$ gives all of $\mathfrak{u}(4)$.

\subsubsection{Complete classification of 2-universal 2-qubit Hamiltonians}

Combining the result of the previous section with Corollary 2 gives the following theorem.

Theorem 2. A two-qubit Hamiltonian $H$ is 2-universal if and only if it does not satisfy any of the following conditions:

1. $H$ is $T$-similar to a local Hamiltonian,

2. $H$ shares an eigenvector with $T$, the gate that swaps two qubits, or

3. $\operatorname{Tr}(H)=0$.

These conditions are easy to check by computing $\tilde{\Xi}$ and applying Corollary 2 .

\section{3-non-universal Hamiltonians}

It turns out that there are 2-non-universal 2-qubit Hamiltonians that are nevertheless 3-universal. In fact, numerical evidence suggests that almost any traceless 2-non-universal Hamiltonian is 3-universal. We do not know a complete characterization of 3-universal 2-qubit Hamiltonians. However, the following are sufficient conditions for a 2-qubit Hamiltonian to be 3-non-universal.

Lemma 9. A 2-qubit Hamiltonian $H$ is 3-non-universal if any of the following conditions holds:

1. $H$ is a local Hamiltonian,

2. $H$ has an eigenvector of the form $|a\rangle|a\rangle$ for some $|a\rangle \in \mathbb{C}^{2}$,

3. $\operatorname{Tr}(H)=0$,

4. $H=r I_{4}+(U \otimes U) A(U \otimes U)^{\dagger}$ for some $r \in \mathbb{R}, U \in \mathrm{U}(2)$, and some antisymmetric Hamiltonian $A \in \mathfrak{u}(4)$ ( $A$ is antisymmetric if $A^{\mathrm{\top}}=-A$ ),

5. $[H, U \otimes U]=0$ for some $U \in \mathrm{U}(2)$ with distinct eigenvalues.

It is easy to see that these Hamiltonians are indeed 3-non-universal. In fact, they are also $n$-non-universal for all $n \geq 3$. Therefore, if one could show that this list is complete, then it would provide a complete characterization of $n$-universal 2-qubit Hamiltonians.

Recall that a 3-non-universal 2-qubit Hamiltonian is also 2-non-universal (see Lemma 2). For each family $\mathcal{F}_{3}$ of 3-non-universal Hamiltonians in Lemma 9 there is a family $\mathcal{F}_{2}$ of 2-non-universal Hamiltonians from Theorem 2 sucht that $\mathcal{F}_{3} \subseteq \mathcal{F}_{2}$ (see Figure 1).

\section{Open problems}

The main result of this paper is a complete characterization of 2-universal 2-qubit Hamiltonians, as summarized in Theorem 2. Several variants of the problem that remain open: 


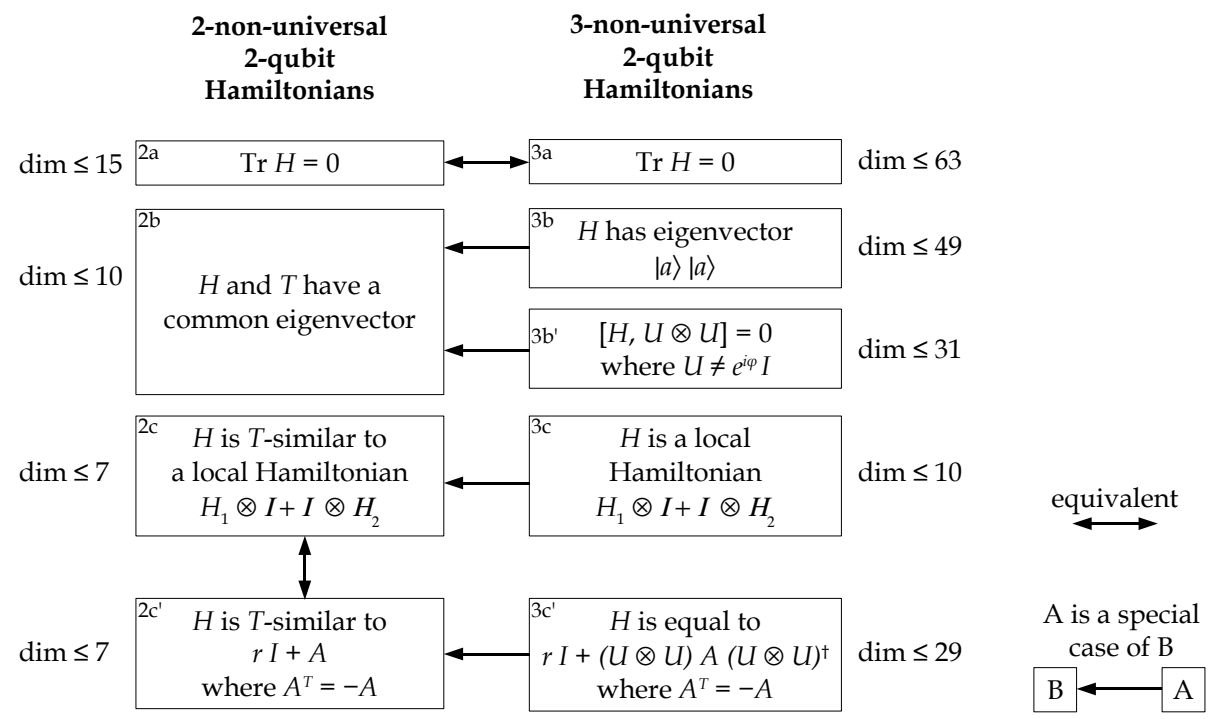

Figure 1: Relations between the families of 2-non-universal and 3-non-universal Hamiltonians. For each family $\mathcal{F}$ we give the maximum achievable value of $\operatorname{dim} \mathcal{L}(H, T H T)$ for $H \in \mathcal{F}$.

1. Which 2-non-universal 2-qubit Hamiltonians are $n$-universal, i.e., become universal on $n \geq 3$ qubits? Also, is there $n_{0} \in \mathbb{N}$ such that $n_{0}$-non-universality implies $n$-non-universality for all $n \geq n_{0}$ ? In particular, is $n_{0}=3$ ?

2. Which 2-qubit Hamiltonians are universal with ancillae? (See Section 2.3.1 and Definition 5 for the definition of universality with ancillae for unitary gates.) One might also consider a scenario in which the number of allowed ancillary qubits is restricted.

3. Which 2-qubit Hamiltonians give us encoded universality, e.g., generate $\mathrm{O}(4)$ ? This question is relevant since the full power of quantum computation can be achieved even with a restricted repertoire of gates. For example, real gates are sufficient [3, 19] since $\mathrm{O}\left(2 \cdot 2^{n}\right)$ contains $\mathrm{U}\left(2^{n}\right)$. We can say that $H$ is $n$-universal in an encoded sense if there exists $k \in \mathbb{N}$ (possibly depending on $n$ ) such that the Lie algebra generated by $H$ on $n+k$ qubits, $\mathcal{L} \subseteq \mathfrak{u}\left(2^{n+k}\right)$, contains $\mathfrak{u}\left(2^{n}\right)$ as a subalgebra. However, it is not even clear how to check this for a particular Hamiltonian.

\section{Acknowledgments}

We thank John Watrous for helpful discussions. Support for this research was provided by MITACS, NSERC, QuantumWorks, and the US ARO/DTO. DL also acknowledges support from CRC, CFI, ORF, and CIFAR.

\section{A Positive time evolution is sufficient}

Claim 1. Let $H \in \mathfrak{u}(N)$ be a Hamiltonian and let $\tau<0$. Then for all $\varepsilon>0$ there exists $t>0$ such that $\left\|e^{-i H \tau}-e^{-i H t}\right\|_{\infty}<\varepsilon$.

Proof. Let $U:=e^{-i H}$. Consider the sequence $\mathcal{K}:=\left\{U^{i}\right\}_{i=1}^{\infty} \subset \mathrm{M}_{N}(\mathbb{C})$. Note that we can think of $\mathrm{M}_{N}(\mathbb{C})$ as a real vector space of dimension $2 N^{2}$. Since $\mathcal{K}$ is bounded with respect to the spectral norm, by the Bolzano-Weierstrass theorem, $\mathcal{K}$ has a convergent subsequence. It follows that for all $\varepsilon>0$ and all $n_{0} \in \mathbb{N}$ there exist $j, k \in \mathbb{N}$ such that $j-k>n_{0}$ and $\varepsilon>\left\|U^{k}-U^{j}\right\|_{\infty}=\left\|I_{N}-U^{j-k}\right\|_{\infty}$. Equivalently, for all $\varepsilon>0$ and all $n_{0} \in \mathbb{N}$ there exists $n>n_{0}$ such that $\varepsilon>\left\|I_{N}-U^{n}\right\|_{\infty}$. Therefore, given $\tau<0$, for all $\varepsilon>0$ there exists $n>|\tau|$ such that

$$
\varepsilon>\left\|I_{N}-e^{-i H n}\right\|_{\infty}=\left\|e^{-i H \tau}-e^{-i H(n+\tau)}\right\|_{\infty} .
$$


Taking $t:=n+\tau>0$, the claim follows.

\section{B Basic properties of the $T$ gate}

In this appendix we restate and prove the basic properites of the $T$ gate introduced in Section 3.1 .

Fact 1. The singlet $|s\rangle$ is an eigenvector of a normal matrix $N \in \mathrm{M}_{4}(\mathbb{C})$ if and only if $[N, T]=0$.

Proof. Suppose $|s\rangle$ is an eigenvector of $N$. Then $\mathcal{B}=\left\{|s\rangle,\left|n_{1}\right\rangle,\left|n_{2}\right\rangle,\left|n_{3}\right\rangle\right\}$ is an orthonormal eigenbasis of $N$ for some orthonormal vectors $\left\{\left|n_{i}\right\rangle\right\}_{i=1}^{3} \subset \mathbb{C}^{4}$. Since $\mathcal{B}$ is orthonormal, $\left\{\left|n_{i}\right\rangle\right\}_{i=1}^{3} \in E_{-}^{\perp}=E_{+}$. Therefore, $\mathcal{B}$ is also an eigenbasis of $T$, and both $N$ and $T$ are simultaneously diagonal in this basis. Thus $[N, T]=0$.

Conversely, suppose $[N, T]=0$. Then $N$ and $T$ are simultaneously diagonal in some orthonormal basis $\mathcal{B}$. Since $|s\rangle$ spans the one-dimensional eigenspace $E_{-}$of $T$, we know that $e^{i \phi}|s\rangle \in \mathcal{B}$ for some $\phi \in \mathbb{R}$. Thus, $|s\rangle$ is an eigenvector of $N$.

Fact 2. A normal matrix $N \in \mathrm{M}_{4}(\mathbb{C})$ has a common eigenvector with the $T$ gate if and only if it has an eigenvector orthogonal to $|s\rangle$.

Proof. The "if" direction is trivial. Conversely, suppose $N$ shares an eigenvector $|v\rangle$ with the $T$ gate. If $|v\rangle \in E_{+}$we are done. Otherwise, $|v\rangle \in E_{-}$, so $|v\rangle=|s\rangle$. Then $\operatorname{span}_{\mathbb{C}}(|v\rangle)^{\perp}=E_{+}$is an invariant subspace of $N$ and it contains an eigenvector of $N$.

Fact 3. Suppose $U \in \mathrm{U}(4)$ and $[U, T]=0$. Then the singlet state $|s\rangle$ is an eigenvector of both $U$ and $U^{\dagger}$.

Proof. Since $[U, T]=0$, we know that $U$ and $T$ are simultaneously diagonal in some orthonormal basis. The singlet $|s\rangle$ must belong to this basis, since it spans the one-dimensional eigenspace $E_{-}$of the $T$ gate. Therefore, $|s\rangle$ has to be an eigenvector of $U$ as well. Note that $U$ and $U^{\dagger}$ have the same eigenvectors. Thus, $|s\rangle$ is also an eigenvector of $U^{\dagger}$.

Fact 5. If a 2-qubit Hamiltonian $H$ has a degenerate eigenvalue, then it shares an eigenvector with $T$ and hence is not universal.

Proof. Suppose $H$ has a degenerate eigenvalue, and let $E$ denote the corresponding eigenspace. Recall that the $T$ gate has a 3 -dimensional (+1)-eigenspace $E_{+}$. Now note that the intersection $E \cap E_{+}$is at least 1-dimensional, since $E, E_{+} \subseteq \mathbb{C}^{4}$ and $\operatorname{dim}(E) \geq 2, \operatorname{dim}\left(E_{+}\right)=3$. Any nonzero $|v\rangle \in E \cap E_{+}$is a common eigenvector of $H$ and the $T$ gate. By Fact 4 we conclude that $H$ is non-universal.

\section{T-similarity to a local Hamiltonian}

In this appendix we prove a result characterizing Hamiltonians that are $T$-similar to some local Hamiltonian, as stated in Section 3.3 . Our proof makes use of the following general characterization of $T$-similarity:

Theorem 3. Hamiltonians $H$ and $H^{\prime}$ are $T$-similar if and only if there exist orthonormal eigenbases $\left\{\left|v_{1}\right\rangle,\left|v_{2}\right\rangle,\left|v_{3}\right\rangle,\left|v_{4}\right\rangle\right\}$ of $H$ and $\left\{\left|w_{1}\right\rangle,\left|w_{2}\right\rangle,\left|w_{3}\right\rangle,\left|w_{4}\right\rangle\right\}$ of $H^{\prime}$ such that $\left\langle v_{i}|H| v_{i}\right\rangle=\left\langle w_{i}\left|H^{\prime}\right| w_{i}\right\rangle$ and $\left|\left\langle v_{i} \mid s\right\rangle\right|=$ $\left|\left\langle w_{i} \mid s\right\rangle\right|$ for all $i \in\{1,2,3,4\}$, where $|s\rangle$ is the singlet state defined in equation (12).

Proof. Assume $H$ and $H^{\prime}$ are $T$-similar, i.e., $H^{\prime}=U H U^{\dagger}$ for some $U \in \mathrm{U}(4)$ with $[U, T]=0$. Since $[U, T]=0$, by Fact 3 we know that $|s\rangle$ is an eigenvector of $U^{\dagger}$. Let $|v\rangle$ be an eigenvector of $H$. Then $U|v\rangle$ is the corresponding eigenvector of $U H U^{\dagger}$. Now we have $|\langle s|(U|v\rangle)|=\mid\left(U^{\dagger}|s\rangle\right)^{\dagger}|v\rangle|=|\langle s \mid v\rangle \mid$, i.e., the corresponding eigenvectors of $H$ and $U H U^{\dagger}$ have the same overlaps with the singlet state. Since conjugation does not change the eigenvalues, the "only if" direction of the theorem follows.

Conversely, assume that $H$ and $H^{\prime}$ have orthonormal eigenbases $\left\{\left|v_{i}\right\rangle\right\}$ and $\left\{\left|w_{i}\right\rangle\right\}$, respectively, with $\lambda_{i}:=\left\langle v_{i}|H| v_{i}\right\rangle=\left\langle w_{i}\left|H^{\prime}\right| w_{i}\right\rangle$ and $r_{i}:=\left|\left\langle v_{i} \mid s\right\rangle\right|=\left|\left\langle w_{i} \mid s\right\rangle\right|$. We can express the singlet state $|s\rangle$ in the 
eigenbases of $H$ and $H^{\prime}$ as follows:

$$
\begin{aligned}
|s\rangle & =\sum_{j=1}^{4} r_{j} e^{i \alpha_{j}}\left|v_{j}\right\rangle \\
& =\sum_{j=1}^{4} r_{j} e^{i \beta_{j}}\left|w_{j}\right\rangle,
\end{aligned}
$$

where $\alpha_{j}, \beta_{j} \in \mathbb{R}$. Now let

$$
U:=\sum_{j=1}^{4} e^{i\left(\beta_{j}-\alpha_{j}\right)}\left|w_{j}\right\rangle\left\langle v_{j}\right| .
$$

We claim that (a) $U H U^{\dagger}=H^{\prime}$ and (b) $|\langle s|U| s\rangle|=1$.

(a) By expressing $U$ as in (43), we have

$$
\begin{aligned}
U H U^{\dagger} & =\sum_{j=1}^{4} e^{i\left(\beta_{j}-\alpha_{j}\right)}\left|w_{j}\right\rangle\left\langle v_{j}\left|\sum_{k=1}^{4} \lambda_{k}\right| v_{k}\right\rangle\left\langle v_{k}\left|\sum_{l=1}^{4} e^{-i\left(\beta_{l}-\alpha_{l}\right)}\right| v_{l}\right\rangle\left\langle w_{l}\right| \\
& =\sum_{k=1}^{4} e^{i\left(\beta_{k}-\alpha_{k}\right)} \lambda_{k} e^{-i\left(\beta_{k}-\alpha_{k}\right)}\left|w_{k}\right\rangle\left\langle w_{k}\left|=\sum_{k=1}^{4} \lambda_{k}\right| w_{k}\right\rangle\left\langle w_{k}\right|=H^{\prime} .
\end{aligned}
$$

(b) By expressing $\langle s|$ as in 42,,$|s\rangle$ as in 41, and $U$ as in 433, we get

$$
\begin{aligned}
\langle s|U| s\rangle & =\sum_{j=1}^{4} r_{j} e^{-i \beta_{j}}\left\langle w_{j}\left|\sum_{k=1}^{4} e^{i\left(\beta_{k}-\alpha_{k}\right)}\right| w_{k}\right\rangle\left\langle v_{k}\left|\sum_{l=1}^{4} r_{l} e^{i \alpha_{l}}\right| v_{l}\right\rangle \\
& =\sum_{k=1}^{4} r_{k} e^{-i \beta_{k}} e^{i\left(\beta_{k}-\alpha_{k}\right)} r_{k} e^{i \alpha_{k}}=\sum_{k=1}^{4} r_{k}^{2}=1
\end{aligned}
$$

Part (a) tells us that $H$ and $H^{\prime}$ are similar via $U$. From (b) it follows that $|s\rangle$ is an eigenvector of $U$, so by Fact 1. $U$ commutes with $T$. Hence $H$ and $H^{\prime}$ are $T$-similar.

Now we can prove the result stated in Section 3.3 characterizing $T$-similarity to a local Hamiltonian:

Lemma 5. A 2-qubit Hamiltonian is $T$-similar to a local Hamiltonian if and only if it has an orthonormal basis of eigenvectors $\left|v_{1}\right\rangle,\left|v_{2}\right\rangle,\left|v_{3}\right\rangle,\left|v_{4}\right\rangle$ corresponding to the eigenvalues $\lambda_{1}, \lambda_{2}, \lambda_{3}, \lambda_{4}$ so that

1. $\left|\left\langle v_{1} \mid s\right\rangle\right|=\left|\left\langle v_{2} \mid s\right\rangle\right|$ and $\left|\left\langle v_{3} \mid s\right\rangle\right|=\left|\left\langle v_{4} \mid s\right\rangle\right|$, and

2. $\lambda_{1}+\lambda_{2}=\lambda_{3}+\lambda_{4}$,

where $|s\rangle$ is the singlet state defined in equation 12 .

Proof. First we prove the "only if" direction. Suppose $H$ is $T$-similar to some local Hamiltonian $H^{\prime}=$ $H_{1} \otimes I+I \otimes H_{2}$. By Theorem 3 it suffices to show that the spectrum of $H^{\prime}$ has the form described in the lemma. We diagonalize $H_{1}$ and $H_{2}$ as follows:

$$
H_{1}=\alpha_{1}\left|v_{1}\right\rangle\left\langle v_{1}\left|+\alpha_{2}\right| v_{2}\right\rangle\left\langle v_{2}\left|, \quad H_{2}=\beta_{1}\right| w_{1}\right\rangle\left\langle w_{1}\left|+\beta_{2}\right| w_{2}\right\rangle\left\langle w_{2}\right| .
$$

Let the first eigenvectors of $H_{1}$ and $H_{2}$ be

$$
\left|v_{1}\right\rangle=\left(\begin{array}{l}
a \\
b
\end{array}\right), \quad\left|w_{1}\right\rangle=\left(\begin{array}{l}
c \\
d
\end{array}\right) .
$$


Since we can ignore the global phase of each eigenvector, we may assume that

$$
\left|v_{2}\right\rangle=\left(\begin{array}{c}
-b^{*} \\
a^{*}
\end{array}\right), \quad\left|w_{2}\right\rangle=\left(\begin{array}{c}
-d^{*} \\
c^{*}
\end{array}\right) .
$$

Then

$$
\left|v_{1}\right\rangle \otimes\left|w_{1}\right\rangle, \quad\left|v_{2}\right\rangle \otimes\left|w_{2}\right\rangle, \quad\left|v_{1}\right\rangle \otimes\left|w_{2}\right\rangle, \quad\left|v_{2}\right\rangle \otimes\left|w_{1}\right\rangle
$$

are eigenvectors of $H^{\prime}$. Calculating the overlaps with $|s\rangle$, we find

$$
\begin{aligned}
& \left|\left\langle s \mid v_{1}, w_{1}\right\rangle\right|^{2}=\frac{1}{2}|a d-b c|^{2}=: r, \\
& \left|\left\langle s \mid v_{2}, w_{2}\right\rangle\right|^{2}=\frac{1}{2}\left|a^{*} d^{*}-b^{*} c^{*}\right|^{2}=\frac{1}{2}|a d-b c|^{2}=r, \\
& \left|\left\langle s \mid v_{1}, w_{2}\right\rangle\right|^{2}=\frac{1}{2}\left|a c^{*}+b d^{*}\right|^{2}=: t, \\
& \left|\left\langle s \mid v_{2}, w_{1}\right\rangle\right|^{2}=\frac{1}{2}\left|-a^{*} c-b^{*} d\right|^{2}=\frac{1}{2}\left|a c^{*}+b d^{*}\right|^{2}=t .
\end{aligned}
$$

The corresponding eigenvalues of $H^{\prime}$ are

$$
\lambda_{1}=\alpha_{1}+\beta_{1}, \quad \lambda_{2}=\alpha_{2}+\beta_{2}, \quad \lambda_{3}=\alpha_{1}+\beta_{2}, \quad \lambda_{4}=\alpha_{2}+\beta_{1},
$$

respectively; they satisfy $\lambda_{1}+\lambda_{2}=\lambda_{3}+\lambda_{4}$. This establishes the "only if" direction.

Now let us prove the "if" direction. For any $H$ with a spectrum satisfying the conditions of the lemma, we construct a local Hamiltonian $H^{\prime}=H_{1} \otimes I+I \otimes H_{2}$ that is $T$-similar to $H$. As before, let $\alpha_{i}$ and $\left|v_{i}\right\rangle$ denote corresponding eigenvalues and eigenvectors of $H_{1}$, and let $\beta_{i}$ and $\left|w_{i}\right\rangle$ denote eigenvalues and eigenvectors of $H_{2}$. In terms of the eigenvalues $\lambda_{i}$ of $H$, we choose the eigenvalues of $H_{1}$ and $H_{2}$ as follows: $\alpha_{1}=0, \alpha_{2}=\lambda_{2}-\lambda_{3}, \beta_{1}=\lambda_{1}$, and $\beta_{2}=\lambda_{3}$. With this choice, the eigenvalues of $H^{\prime}$ are

$$
\alpha_{1}+\beta_{1}=\lambda_{1}, \quad \alpha_{2}+\beta_{2}=\lambda_{2}, \quad \alpha_{1}+\beta_{2}=\lambda_{3}, \quad \alpha_{2}+\beta_{1}=\lambda_{4},
$$

where the last equality holds since $\lambda_{1}+\lambda_{2}=\lambda_{3}+\lambda_{4}$. It remains to choose eigenvectors of $H_{1}$ and $H_{2}$ to obtain the required overlaps with $|s\rangle$. Notice that $\left|v_{1}\right\rangle$ and $\left|w_{1}\right\rangle$ completely determine the overlaps, since without loss of generality we can take $\left|v_{2}\right\rangle$ and $\left|w_{2}\right\rangle$ as in (50). In fact, it suffices to choose $\left|v_{1}\right\rangle,\left|w_{1}\right\rangle \in \mathbb{R}^{2}$. If the angle between real unit vectors $\left|v_{1}\right\rangle=\left(\begin{array}{c}a \\ b\end{array}\right)$ and $\left|w_{1}\right\rangle=\left(\begin{array}{l}c \\ d\end{array}\right)$ is $\theta$, then $a d-b c=\sin \theta$ and $a c+b d=\cos \theta$. Thus, the overlaps (52) and (54) are $\frac{1}{2} \sin ^{2} \theta=r$ and $\frac{1}{2} \cos ^{2} \theta=t$, respectively. Therefore, we can take any two real unit vectors having angle $\theta=\arcsin \sqrt{2 r}$. Since $H$ and $H^{\prime}$ satisfy the conditions of Theorem 3 , they are $T$-similar.

\section{Almost all two-qubit unitaries are 2-universal}

In this paper, we have primarily focused on universality of Hamiltonians. In this appendix, we return to the question of universality of unitary gates. In particular, we show how our results easily imply that a Haar-uniform 2-qubit unitary is almost surely universal.

Definition 10. We say that a 2-qubit unitary $U$ is 2-universal if, for any $V \in \mathrm{U}(4)$ and any $\varepsilon>0$, there exist $l \in \mathbb{N}$ and $N_{1}, \ldots, N_{l} \in \mathbb{N} \cup\{0\}$ such that

$$
\left\|V-U^{N_{l}} \ldots U^{N_{3}}(T U T)^{N_{2}} U^{N_{1}}\right\|_{\infty}<\varepsilon .
$$

We say that a Hamiltonian $H$ corresponds to a unitary $U$ if $e^{-i H}=U$. In general, for a given $U$, such Hamiltonian is not unique (if $H$ corresponds to $U$ then so does $H+2 \pi k|v\rangle\langle v|$ for any integer $k$ and any eigenvector $|v\rangle$ of $H$ ). However, if we also demand that all eigenvalues of $H$ are in the interval $(-\pi, \pi]$, then there is a unique such Hamiltonian.

If $\lambda_{1}, \ldots, \lambda_{4}$ are the eigenvalues of $U \in \mathrm{U}(4)$, we can associate to each $\lambda_{j}$ a unique phase $\theta_{j} \in(-\pi, \pi]$ such that

$$
\lambda_{j}=e^{-i \theta_{j}} .
$$


We call these the canonical phases of $U$. Furthermore, if the eigenvalues $\lambda_{1}, \ldots, \lambda_{4}$ of $U$ are distinct, we can associate to each $\lambda_{j}$ a unique eigenvector $\left|v_{j}\right\rangle$ such that $U=\sum_{j=1}^{4} \lambda_{j}\left|v_{j}\right\rangle\left\langle v_{j}\right|$. We call

$$
H:=\sum_{j=1}^{4} \theta_{j}\left|v_{j}\right\rangle\left\langle v_{j}\right|
$$

the canonical Hamiltonian corresponding to $U$. Note from Lemma 10 below that, for a Haar-random $U$, all $\theta_{j}$ are almost surely distinct, in which case there is no freedom in choosing the eigenvectors $\left|v_{j}\right\rangle$.

Building on our characterization of 2-universal Hamiltonians, we now show that a Haar-random unitary matrix $U \in \mathrm{U}(4)$ is almost surely 2-universal. The main idea is that the canonical Hamiltonian $H$ corresponding to such $U$ is almost surely 2-universal and, moreover, natural powers of $U$ can be used to approximate the evolution according to $H$ for any amount of time. To achieve the latter, we need to ensure that the eigenvalues of $H$ are rationally independent with probability one. Recall that numbers $\alpha_{1}, \ldots, \alpha_{n} \in \mathbb{R}$ are rationally dependent if $\sum_{i=1}^{n} q_{i} \alpha_{i}=0$ for some $q_{1}, \ldots, q_{n} \in \mathbb{Q}$, not all of which are zero.

Lemma 10. Let $\theta_{1}, \theta_{2}, \theta_{3}, \theta_{4} \in(-\pi, \pi]$ be the canonical phases of a Haar-random unitary $U \in \mathrm{U}(4)$. Then with probability one the angles $\pi, \theta_{1}, \theta_{2}, \theta_{3}, \theta_{4}$ are rationally independent.

Proof. The joint density of eigenvalues of a Haar-random unitary was obtained by Weyl and is given by

$$
f\left(\theta_{1}, \theta_{2}, \theta_{3}, \theta_{4}\right):=\frac{1}{(2 \pi)^{4} 4 !} \prod_{j<k}\left|e^{i \theta_{j}}-e^{i \theta_{k}}\right|^{2}
$$

(see e.g. [10]). For the purpose of this proof the specific form of $f\left(\theta_{1}, \theta_{2}, \theta_{3}, \theta_{4}\right)$ is irrelevant; we will only use the fact that $f\left(\theta_{1}, \theta_{2}, \theta_{3}, \theta_{4}\right)$ is upper bounded by some fixed finite constant.

Consider the set $(-\pi, \pi]^{4}$ of all possible canonical phases of a $4 \times 4$ unitary. Let us exclude from this set all those points $\vec{\theta}:=\left(\theta_{1}, \theta_{2}, \theta_{3}, \theta_{4}\right)$ for which $\pi, \theta_{1}, \theta_{2}, \theta_{3}, \theta_{4}$ are rationally dependent, i.e., $q_{0} \pi+\sum_{i=1}^{4} q_{i} \theta_{i}=0$ for some $q_{0}, q_{1}, \ldots, q_{4} \in \mathbb{Q}$. To account for all possible rational dependences, for each choice of coefficients $\vec{q}:=\left(q_{0}, q_{1}, \ldots, q_{4}\right) \in \mathbb{Q}^{5}$, we define an affine hyperplane

$$
A_{\vec{q}}:=\left\{\vec{\theta} \in \mathbb{R}^{4}: q_{0} \pi+\sum_{i=1}^{4} q_{i} \theta_{i}=0\right\}
$$

consisting of all those angles $\vec{\theta}$ that are rationally dependent and their dependence can be expressed with coefficients given by $\vec{q}$. Note that the set $A_{\vec{q}}$ has zero measure in $\mathbb{R}^{4}$, so

$$
A:=\bigcup_{\vec{q} \in \mathbb{Q}^{5}} A_{\vec{q}}
$$

also has zero measure, as it is a countable union of measure-zero sets.

If $S:=(-\pi, \pi]^{4}$ is the set of all canonical phases and $\mu$ denotes the standard Lebesgue measure on $\mathbb{R}^{4}$, the probability that a Haar-random unitary has rationally independent canonical phases is

$$
\int_{S \backslash A} f d \mu
$$

where $f$ is given by equation 61 . However, since the set $S \backslash A$ differs from $S$ only by a set of measure zero, the Lebesgue integral of a bounded function over the two sets is the same [5. p. 40]. Thus,

$$
\int_{S \backslash A} f d \mu=\int_{S} f d \mu=1
$$

and the result follows.

Lemma 11. Let $\vec{\theta}=\left(\theta_{1}, \ldots, \theta_{n}\right) \in(-\pi, \pi]^{n}$ be an $n$-tuple of angles, where each $\theta_{i}$ is irrational with respect to $\pi$. Then for any $\varepsilon>0$ there exists $N \in \mathbb{N}$ such that $0<\left|N \theta_{i} \bmod (-\pi, \pi]\right| \leq \varepsilon$ for all $i \in\{1, \ldots, n\}$. 
Proof. Pick $m:=\lceil 2 \pi / \varepsilon\rceil$ and partition $(-\pi, \pi]$ into $m$ segments of size $2 \pi / m \leq \varepsilon$. For a given $r \in \mathbb{N}$, the components of $r \vec{\theta}$ fall into a combination of these segments. Each such combination can be specified by an element of the finite set $\{1, \ldots, m\}^{n}$. On the other hand, the number of different possible values of $r \in \mathbb{N}$ is infinite. Thus, by the pigeonhole principle, there exist distinct $r, s \in \mathbb{N}$ such that for each $i$, the values $r \theta_{i}$ and $s \theta_{i}$ modulo $2 \pi$ fall in the same segment (which can depend on $i$ ). For each $i$, by the irrationality assumption, $r \theta_{i} \not \equiv s \theta_{i}$ mod $2 \pi$; moreover, both $r \theta_{i}$ and $s \theta_{i}$ fall in the interior of the $(2 \pi / m)$-sized segments. Thus, taking $N:=|r-s|$ ensures that the components of $N \vec{\theta} \bmod (-\pi, \pi]$ are all $\varepsilon$-close to yet distinct from zero.

We are now ready to show that for almost all unitary matrices $U$, we can use their natural powers to simulate the evolution $e^{-i H t}$ according to the canonical Hamiltonian $H$ for any time $t \in \mathbb{R}$.

Lemma 12. Let $U \in \mathrm{U}(4)$ and let $\vec{\theta}=\left(\theta_{1}, \theta_{2}, \theta_{3}, \theta_{4}\right) \in(-\pi, \pi]^{4}$ be the canonical phases of $U$. If the values $\theta_{1}, \theta_{2}, \theta_{3}, \theta_{4}, \pi$ are rationally independent then for any $t \in \mathbb{R}$ and any $\varepsilon>0$ there exists $N \in \mathbb{N}$ such that

$$
\left\|U^{N}-e^{-i H t}\right\|_{\infty}<\varepsilon
$$

where $H$ is the canonical Hamiltonian corresponding to $U$.

Proof. Note that

$$
\left\|U^{N}-e^{-i H t}\right\|_{\infty} \leq \sum_{j=1}^{4}\left|e^{i N \theta_{j}}-e^{-i t \theta_{j}}\right| .
$$

Now let $\vec{\phi}=\left(\phi_{1}, \phi_{2}, \phi_{3}, \phi_{4}\right):=-t \vec{\theta} \bmod (-\pi, \pi] \in(-\pi, \pi]^{4}$ and note that

$$
\left|e^{i N \theta_{j}}-e^{i \phi_{j}}\right|=\left|e^{i\left(N \theta_{j}-\phi_{j}\right)}-1\right|=\sqrt{2-2 \cos \left(N \theta_{j}-\phi_{j}\right)} \leq\left|N \theta_{j}-\phi_{j}\right| .
$$

Combining equation (67) and equation 68, we obtain

$$
\left\|U^{N}-e^{-i H t}\right\|_{\infty} \leq\|N \vec{\theta}-\vec{\phi}\|_{1} .
$$

Our goal is to pick $N$ so that $\|N \vec{\theta}-\vec{\phi}\|_{1} \leq \varepsilon$.

By Lemma 11, we can find $M \in \mathbb{N}$ such that each of the components of $\vec{\theta}^{\prime}:=M \vec{\theta} \bmod (-\pi, \pi]$ is $\frac{\varepsilon}{8}$-close to zero. Next, let us consider the linear flow $\Phi\left(\overrightarrow{\theta^{\prime}}, x\right):=x \overrightarrow{\theta^{\prime}} \bmod (-\pi, \pi]$ on the 4 -torus $(-\pi, \pi]^{4}$. Since the values $\theta_{1}^{\prime}, \theta_{2}^{\prime}, \theta_{3}^{\prime}, \theta_{4}^{\prime}, \pi$ are rationally independent, the flow $\Phi\left({\overrightarrow{\theta^{\prime}}}^{\prime} x\right)$ is dense in $(-\pi, \pi]^{4}[12$. Thus we can find $x \in \mathbb{R}$ such that $\left\|\vec{\phi}-\Phi\left(\overrightarrow{\theta^{\prime}}, x\right)\right\|_{1}<\frac{\varepsilon}{2}$. Furthermore, we have $\left\|\Phi\left(\overrightarrow{\theta^{\prime}}, x\right)-\Phi\left(\overrightarrow{\theta^{\prime}},\lfloor x\rfloor\right)\right\|_{1}=\left\|\overrightarrow{\theta^{\prime}}\right\|_{1}|x-\lfloor x\rfloor|<\frac{\varepsilon}{2}$, since the four components of $\overrightarrow{\theta^{\prime}}$ are all $\frac{\varepsilon}{8}$-close to zero. Combining the last two inequalities yields $\left\|\vec{\phi}-\Phi\left(\overrightarrow{\theta^{\prime}},\lfloor x\rfloor\right)\right\|_{1}<\varepsilon$. Therefore, taking $N:=M\lfloor x\rfloor$ ensures that $\|N \vec{\theta}-\vec{\phi}\|_{1}<\varepsilon$ and hence $\left\|U^{N}-e^{-i H t}\right\|_{\infty}<\varepsilon$ as desired.

We are now ready to show that almost all 2-qubit unitaries are universal.

Theorem 4. A Haar-random unitary $U \in \mathrm{U}(4)$ is almost surely 2-universal.

Proof. By Lemma 10, the canonical phases $\theta_{1}, \theta_{2}, \theta_{3}, \theta_{4} \in(-\pi, \pi]$ of $U$ are almost surely rationally independent. Hence, it suffices to prove the theorem statement for such unitaries $U$.

Applying Lemma 12 to such unitaries $U$, we can use natural powers of $U$ to simulate the evolution according to its corresponding canonical Hamiltonian $H$ for any time $t \in \mathbb{R}$. So to establish the theorem it remains to argue that $H$ is 2-universal. We do this by showing that $H$ does not satisfy any of the three conditions in Theorem 2 If $H$ is $T$-similar to a local Hamiltonian then $\theta_{i}+\theta_{j}=\theta_{k}+\theta_{l}$ for $\{i, j, k, l\}=$ $\{1, \ldots, 4\}$. Similarly, if $\operatorname{Tr}(H)=0$ then $\sum_{i=1}^{4} \theta_{i}=0$. Therefore, each of the conditions (1) and (3) implies that the $\theta_{i}$ are rationally dependent, which contradicts our initial assumption. Let us now examine condition (2). If $H$ shares an eigenvector with $T$ then, by Fact $2, H$ (and hence also $U$ ) has an eigenvector orthogonal to the singlet state $|s\rangle=\frac{1}{\sqrt{2}}(|01\rangle-|10\rangle)$. The probability that a Haar-random $U$ has an eigenvector orthogonal to $|s\rangle$ is zero. Therefore, $H$ almost surely does not satisfy any of the three conditions and is thus 2-universal. 
This provides an alternate proof of the main result of [8, 14, avoiding the shortcomings discussed in Section 2.3.3. However, unlike in our main result on Hamiltonian universality (Theorem 21), we have not characterized precisely which 2-qubit unitaries are universal. To the best of our knowledge, it remains open to find such a characterization.

\section{References}

[1] Adriano Barenco. A universal two-bit gate for quantum computation. Proceedings of the Royal Society of London A, Mathematical and Physical Sciences, 449(1937):679-683, 1995. arXiv:quant-ph/9505016.

[2] Adriano Barenco, Charles H. Bennett, Richard Cleve, David P. DiVincenzo, Norman H. Margolus, Peter W. Shor, Tycho Sleator, John A. Smolin, and Harald Weinfurter. Elementary gates for quantum computation. Physical Review A, 52(5):3457-3467, 1995. arXiv:quant-ph/9503016.

[3] Ethan Bernstein and Umesh Vazirani. Quantum complexity theory. In Proceedings of the 25th Annual ACM Symposium on Theory of Computing, pages 11-20. ACM, 1993.

[4] P. Oscar Boykin, Tal Mor, Matthew Pulver, Vwani Roychowdhury, and Farrokh Vatan. On universal and fault-tolerant quantum computing. In Proceedings of the 40 th Annual IEEE Symposium on Foundations of Computer Science, pages 486-494. IEEE, 1999. arXiv:quant-ph/9906054.

[5] Herald Cramér. Mathematical Methods of Statistics. Princeton University Press, 1999.

[6] George Cybenko. Reducing quantum computations to elementary unitary operations. Computing in Science and Engineering, 3(2):27-32, 2001.

[7] David Deutsch. Quantum computational networks. Proceedings of the Royal Society of London A, Mathematical and Physical Sciences, 425(1868):73-90, 1989.

[8] David Deutsch, Adriano Barenco, and Artur Ekert. Universality in quantum computation. Proceedings of the Royal Society of London A, Mathematical and Physical Sciences, 449(1937):669-677, 1995. arXiv:quant-ph/9505018.

[9] David P. DiVincenzo. Two-bit gates are universal for quantum computation. Physical Review A, 51:1015-1022, 1995. arXiv:cond-mat/9407022.

[10] Fumio Hiai and Denes Petz. The Semicircle Law, Free Random Variables and Entropy. American Mathematical Society, 2006.

[11] Nathan Jacobson. Lie Algebras. Interscience Publishers, 1962.

[12] Anatole Katok and Boris Hasselblatt. Introduction to the Modern Theory of Dynamical Systems. Cambridge University Press, 1997.

[13] Alexei Yu Kitaev. Quantum computations: algorithms and error correction. Russian Mathematical Surveys, 52(6):1191-1249, 1997.

[14] Seth Lloyd. Almost any quantum logic gate is universal. Physical Review Letters, 75(2):346-349, 1995.

[15] Laura Mancinska. Characterization of universal 2-qubit Hamiltonians. Master's thesis, University of Waterloo, 2009.

[16] Michael A. Nielsen and Isaac L. Chuang. Quantum Computation and Quantum Information. Cambridge University Press, 2000.

[17] John Preskill. Fault-tolerant quantum computation. In Hoi-Kwong Lo, Sandu Popescu, and Tim Spiller, editors, Introduction to Quantum Computation and Information, pages 213-269. World Scientific, 1998. arXiv:quant-ph/9712048. 
[18] Michael Reck, Anton Zeilinger, Herbert J. Bernstein, and Philip Bertani. Experimental realization of any discrete unitary operator. Physical Review Letters, 73:58-61, 1994.

[19] Terry Rudolph and Lov K. Grover. A 2 rebit gate universal for quantum computing, 2002. arXiv:quant$\mathrm{ph} / 0210187$.

[20] Peter W. Shor. Fault-tolerant quantum computation. In Proceedings of the 37th Annual IEEE Symposium on Foundations of Computer Science, pages 56-65. IEEE, 1996. arXiv:quant-ph/9605011.

[21] Nik Weaver. On the universality of almost every quantum logic gate. Journal of Mathematical Physics, 41(1):240-243, 2000. 